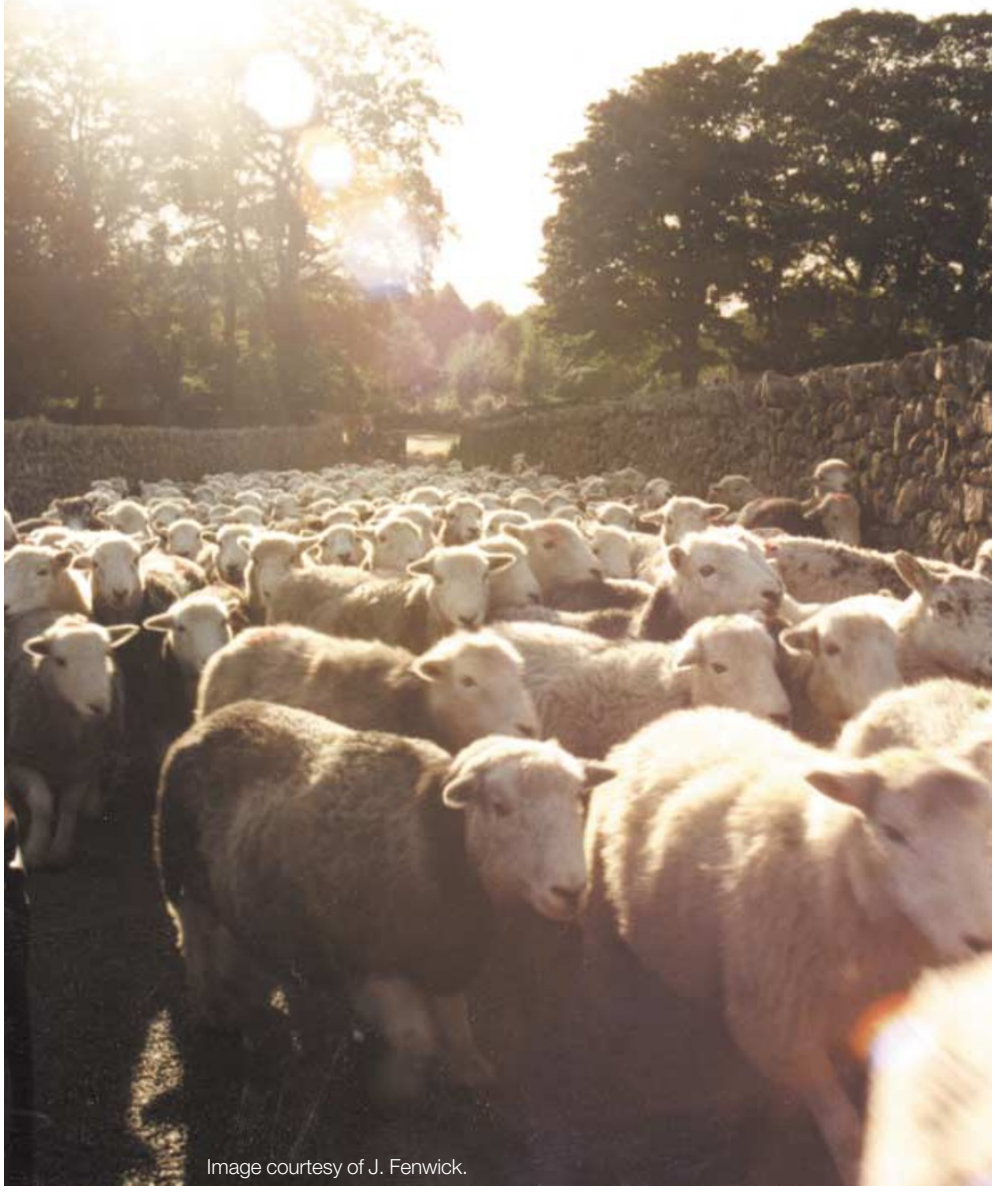

\title{
Blocking the pathway to excitotoxicity
}

Stroke is one of the most common causes of brain damage and disability, and many avenues are being explored in the search for therapeutic advances. One approach is to block the ability of NMDA ( $N$-methyl-D-aspartate) receptors to mediate ischaemic damage, but blocking the receptors themselves is not a feasible strategy. Aarts and colleagues have come up with another option: using small peptides to block the interaction of NMDA receptors with the protein PSD95 (postsynaptic density 95), which couples the receptors to the rest of the postsynaptic machinery and to excitotoxicity.

We know that NMDA receptors mediate excitotoxicity through their interaction with PSD95, and that mutations in PSD95 that block downstream signalling initiated by NMDA receptors can also prevent ischaemic damage. But knocking out or mutating PSD95 is not a useful approach to treating stroke, and blocking NMDA receptors is harmful. However, Aarts et al. reasoned that it might be possible to block the interaction between NMDA receptors and PSD95. They developed a small peptide consisting of the carboxy-terminal nine residues of the NR2B subunit of the NMDA receptor (the domain that binds to PSD95), fused to the cell-membrane transduction domain of the HIV Tat protein (to allow the peptide to enter cells).

In cultures, the fusion peptide entered neurons and reduced the binding of the NR2B subunit to PSD95 without interfering with NMDA receptor currents or calcium influx. It also reduced neuronal death induced by NMDA treatment - even
COGNITIVE CONTROL

\section{The hectic life of the brain}

As I type these words, my phone rings. I answer the call, eventually hang up and restart typing. Now an e-mail arrives and interrupts me again. I read the message, send a reply and continue to write. This ability to adapt my behaviour to satisfy current demands in the presence of competing information is known as 'cognitive control'. A recent paper by Dreher and Berman contributes to our understanding of its neural basis by dissociating the contribution of two different areas of the frontal lobe to cognitive control during a task-switching paradigm.

The authors used a task in which subjects had to report whether a single letter was a consonant or a vowel, lower or upper case, or before or after the letter ' $m$ ' in the alphabet. Which rule applied depended on the colour of the letter (red, green or yellow, respectively), and the authors presented the stimuli in blocks of three letters, one at a time, followed by a period of interruption. For example, one block consisted of a red letter first, then a green one, with a yellow one at the end. After a brief interruption, the next block consisted of a sequence of red, green and red letters. For each letter, the subject had to report its characteristics on the basis of the appropriate rule.

Dreher and Berman asked which brain regions respond when subjects switch to a recently performed task compared with a less recent one. They measured the response time to the last task in a triplet when it had been performed recently (the last 'red' in a block of red, green and red letters), and compared it with the response time to a task that had been performed less recently (the 'yellow' in a red-green-yellow sequence). In this comparison, the response was slower when the task had been performed recently, and there was a specific activation of the lateral prefrontal cortex concomitant with this slower response. This indicates that inhibition of the disengaged task is active for some time and must be overcome to re-engage in the same task. These observations highlight the relevance of the lateral prefrontal cortex to cognitive inhibition, and constitute a rare dissociation between this phenomenon and memory processes.

The authors also investigated the neural basis of re-engagement in a task after an interruption, and found activation of the anterior cingulate cortex during this phenomenon. Behaviourally, re-engagement increased response times and reduced error rates, consistent with the involvement of the anterior cingulate cortex in alertness.

The neural substrates of cognitive control have been hard to pinpoint, and this elegant dissociation and the clever experimental design should stimulate further work in this area. Specifically, it will be important to establish how different regions of the frontal lobe interact to generate the transitions between cognitive operations, which we experience as a seamless continuum.

Juan Carlos López

(2) References and links ORIGINAL RESEARCH PAPER Dreher, J.-C. \& Berman, K. F. Fractionating the neural substrate of cognitive control processes. Proc. Natt Acad. Sci. USA 99, 14595-14600 (2002) FURTHER READING Miller, E. K. The prefrontal cortex and cognitive control. Nature Rev. Neurosci. 1, 59-65 (2000) 
if the peptide was given one hour after the NMDA.

But will this strategy work in vivo? After showing that the peptide could enter the brain when injected intraperitoneally in rats, the authors tested whether it could reduce the harmful effects of transient middle cerebral artery occlusion (MCAO) - a commonly used model of stroke. Injection of the peptide either before or one hour after the onset of MCAO not only reduced the volume of the resulting infarct, but also significantly reduced the severity of neurological symptoms (postural reflex and forelimb placing deficits) caused by the occlusion.

This work presents exciting possibilities for developing new treatments for stroke. Although much work will be needed to ensure that suppressing signalling downstream of the NMDA receptor has no harmful side effects, there is cause for optimism.

Rachel Jones

(2) References and links ORIGINAL RESEARCH PAPER Aarts, M. et al. Treatment of ischemic brain damage by perturbing NMDA receptor-PSD-95 protein interactions. Science 298, 846-850 (2002)

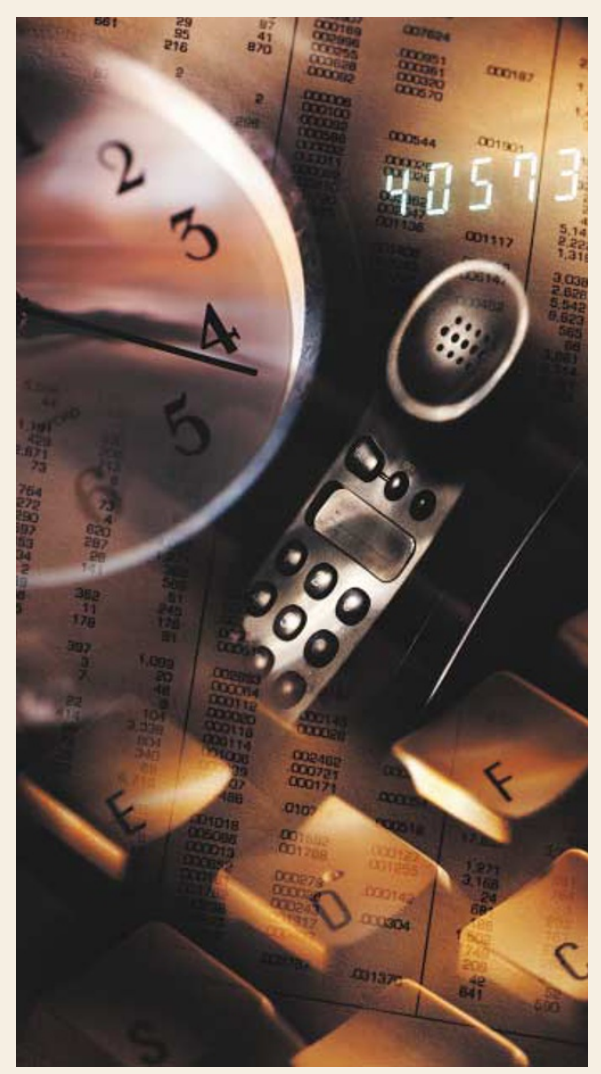

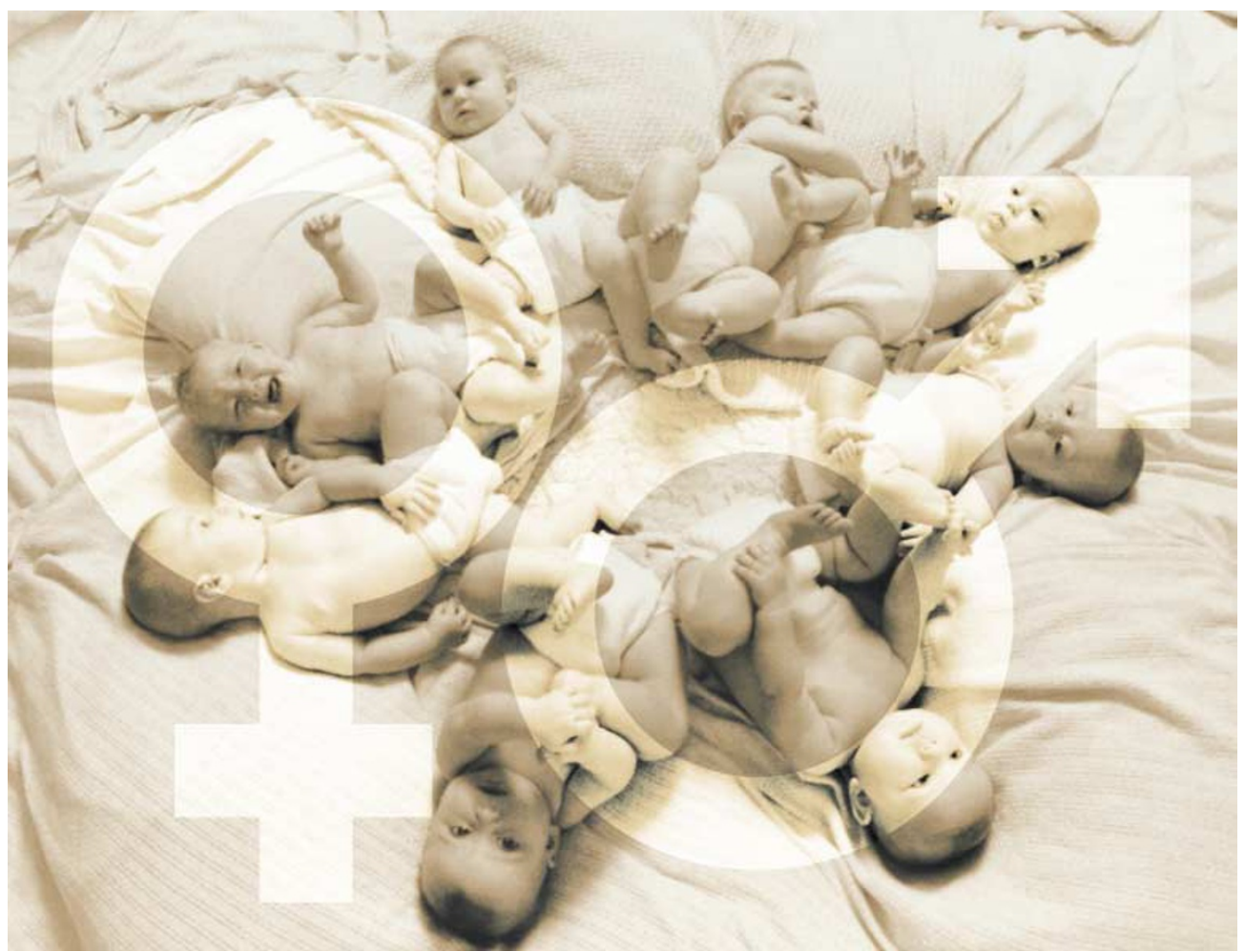

\section{MODEL SYSTEMS}

\section{The genetics of Venus and Mars}

Delving into the minds of men and women can be a profitable enterprise, as the authors of bestsellers such as Men Are from Mars, Women Are from Venus would no doubt testify. Neuroscientists have also taken a keen interest in male-female differences - or dimorphisms - in the brain, but usually to answer more fundamental scientific questions than why men leave the lavatory seat up, or why women take so long to get dressed. In the Journal of Neuroscience, De Vries et al. report on a new transgenic mouse model for studying the genetic basis of sexual dimorphism.

Sexual dimorphism in the brain can take many forms, from variations in the sizes of various structures to differences in behaviour. Sex hormones exert a strong influence on the brain, both during development and throughout life, so it has been assumed that most masculine or feminine brain characteristics are acquired secondary to testis or ovary development. However, De Vries et al. proposed that there might be genes on the sex chromosomes that affect the brain directly, so they created a mouse model that would help them to identify these factors.

Testis development depends on a single gene on the $\mathrm{Y}$ chromosome - the Sry gene. In the absence of $S r y$, ovaries develop by default. The authors generated a transgenic mouse line in which Sry was moved from the $\mathrm{Y}$ chromosome to an autosome. By performing various intercrosses between these and wild-type mice, they obtained XY 'females' (designated $\mathrm{XY}^{-}$), XX' 'males' (XXSry), and XY males with Sry on an autosome instead of the $Y$ chromosome (XY-Sry). They compared XXSry males with $\mathrm{XY}^{-} S r y$ animals rather than wild-type males, to control for the possibility that Sry functions differently in the context of an autosome.

Consistent with previous findings, most structural and behavioural dimorphisms were determined by whether the mouse had testes or ovaries. However, when they compared the density of vasopressin-immunoreactive (VP-ir) fibres in the brains of their mice, they made an intriguing discovery. Normally, males have a higher density of VP-ir fibres in the septum than females, and the $\mathrm{XY}^{-}$Sry mice had a higher density of fibres than XXSry mice, even though the XXSry mice had testes. Similarly, the $\mathrm{XY}^{-}$mice had a higher density of fibres than normal XX mice, even though both had ovaries. These findings indicate that this characteristic is influenced by factors other than Sry on the sex chromosomes.

De Vries et al. speculate that three types of gene could be responsible for non-hormonally induced sexual dimorphisms - genes on the $\mathrm{Y}$ chromosome, $\mathrm{X}$-linked genes that escape $\mathrm{X}$-inactivation, and $\mathrm{X}$-linked genes whose activity is modified by maternal or paternal imprinting. The influence of the sex chromosomes on behaviour has important sociological as well as scientific implications; for example, men with an extra copy of the $\mathrm{Y}$ chromosome show a tendency towards aggressive and criminal behaviour. Is this simply the result of too much testosterone, or would an extra copy of the $\mathrm{Y}$ chromosome minus the Sry gene have a similar effect on behaviour? The new mouse model provides a powerful means of addressing this and other important questions about sexually dimorphic traits.

Heather Wood

(2) References and links

ORIGINAL ReSeARCh PAPER De Vries, G. J. et al. A model system for study of sex chromosome effects on sexually dimorphic neural and behavioural traits. J. Neurosci. 22, 9005-9014 (2002) 